\title{
The Diagnosis of Forging Bevel Gears on the Information Merge of Wavelet Neural Network
}

\author{
Zhanjun Liu \\ Faculty of Aerospace Engineering,Shenyang Aerospace University,Shenyang 110136,China \\ Izjlzjc@163.com
}

Keywords: wavelet neural network; bevel gear ;defect ; information merge ;

\begin{abstract}
The synthetical symptoms of bevel gear is concluded. Using wavelet and mixed data merge does the intelligence diagnosis to the defect of bevel gear, which is integrated with data, characteristic, decision grate and nerve network . A model of wavelet neural network is constructed. In order to reduce no confirm of defect analysis, the excellent diagnosis way is studied with the information of many sources fill and redundant. The result is given that using mixed data merge may raise tolerate character with the help of many sources fill and redundant, and using wavelet and mixed data merge does the effective diagnosis of bevel gear.
\end{abstract}

\section{Introduction}

Artificial neural network is as a kind of information processing technology, adopting the method of program design is completely different with conventional algorithms, it can refer to a large database, to find out the information contained in mode, without having to know what data is important, it is not because the information is not clear or incomplete[1]. It is a parallel distributed processing system, driven by data, the method of knowledge acquisition is from learning on the sample, this kind of learning is not just memorizing some data, but is extracted from the sample containing some of the basic characteristics and laws. These features are very suitable for solving the problems of prior knowledge, and the fault diagnosis is difficult to hunt for. Structurally, the neural network is composed of multiple neurons connected in a certain way and form of nonlinear systems, which in essence is fault tolerant, able to identify with the noise and the change of input pattern, for the change is big, fault processing conditions is complex, it is good to interfere with the serious problem of complex system fault[2]. In addition, no contact network is nodes in the tree, and the layer number of the node number is greater, the overall system is parallel processing, short response time can meet the requirements of real-time diagnosis. Due to the uniqueness of neural network structure and information processing method, it can be used for condition monitoring and fault diagnosis of complex structure system research. In this paper, the network input layer is corresponding from the dynamic seal in the shaft diagnosis object, pressure, temperature, the hardness of alignment, radial runout value, surface roughness to extract the characteristic vector and the output layer is correspond to the diagnosis of all kinds of fault state of objects.

\section{The basic theory of information fusion technology}

Information fusion or data fusion is to test the data from multiple sources, correlation, estimation and comprehensive grade, various processing, in order to obtain the accurate estimate of the state of the object to be tested and evaluated. The advantage of the coordination and joint action is by using multi-sensor to enhance the overall performance of the detection system[3].

Information fusion system is often will divide the data fusion layer, feature fusion layer and decision-making fusion of three levels.Policy makers refers to the fusion before integration, each sensor signal of local processing is done first, namely each sensor and the corresponding processing unit are respectively independently completed the feature extraction and decision tasks, such as correlation is be into the fusion center. Therefore, the essence of this method is based on certain criteria and the credibility of each decision makes optimal decisions. Policy makers fusion is a senior of data fusion fusion. Its advantage is small amount 
of data communication, real-time performance is good, which can handle asynchronous information fusion of different types of information effectively. In one or several sensor failure, the system can still continue to work, which have good fault tolerance, the reliability of the system is high. Therefore, policy makers fusion is a hotspot in the research of the information fusion. However, this technology also has its disadvantages, such as the loss of the original information, the time varying characteristics of the object and prior knowledge acquisition are difficult, the knowledge base of features are such as huge amount and difficult to get the application[4].

In the actual choice, blend mode should be determined by the specific problems. Fault diagnosis is in dynamic sealing lip seal shaft, due to the nature of a variety of testing equipment, for all kinds of detection methods of defect information model is different, it is difficult to directly related to its, so it is not easy for data fusion. By the testing equipment defect features of inconsistency makes using feature layer convergence difficulties, therefore, more practical choice is to fusion decision makers. This way is not only minimal output information constraints on testing equipment, and system reliability is higher[5].

\section{The structure of the wavelet neural network model}

Artificial neural network model have a hopfield net, forward multi-layer neural network and adaptive resonance theory, such as a variety of types model. Most applications in the field is of diagnosis, the most effective is the forward multi-layer neural network model[6]. Because the network model is used in the process of learning training after the error propagation algorithm, it is also called the BP network. BP network is all neurons within the layers of adjacent layer, neurons unavailability of bonding is strength value. Weight by known is to input and output information of the training sample, to adjust the network training and learning. This kind of adjustment is by BP algorithm. BP algorithm is a kind of error correction model learning algorithm, which is composed of forward and reverse transmission process. In the forward propagation, the input information is from the input layer, through the role function, which is step by step to the hidden layer and output layer, if it can not get the desired output in the layer, then it came into the back propagation process, the error information is connecting path along the original return, by modifying the weights between neurons in each layer, make the output error is minimum. $\mathrm{M}$ input mapping of wavelet network model is as follows :

$$
f(X)=\sum_{i=1}^{n} w_{i} \Phi_{i}\left(\frac{X-b_{i}}{a_{i}}\right)
$$

The wavelet basis function network is to select Morlet wavelet. The form of wavelet for:

$$
\psi(x)=e^{-\frac{x^{2}}{2}} \cos (5 x)
$$

The corresponding discrete form as follows:

$$
\Phi_{m n}(x)=a_{0}^{-m / 2} \Phi\left(a_{0}^{-m} x-n b_{0}\right)
$$

With wavelet basis function, it also needs through time-frequency analysis to determine the $\mathrm{m}$, $\mathrm{n}$. Setting can be used to support.

$$
\left\|f_{t}\right\|^{2}=\frac{1}{P} \sum_{i=1}^{P} y_{i}^{2} \bullet \Delta x_{i},\left(\Delta x_{i}=x_{i}-x_{i-1}\right)
$$

Set energy is estimated to be in, there are: 


$$
Q_{\varepsilon}^{+}(f)=\left[x_{\min }, x_{\max }\right] \cdot\left[\omega_{\min }^{*}, \omega_{\max }^{*}\right]
$$

With time and frequency region, it can determine the wavelet base. So called determined wave is to determine the wavelet base vector $\Phi$, and values range. By these results is given in literature, for each identified is values range[7].

\section{Metal flow in cold forging of bevel gears}

The material flow during bevel gear forming is initially axisymmetric until the flow front touches the die at the tooth root. Then the flow near the root is diverted into the tooth and becomes three dimensional. However, due to symmetry conditions in planes through the tooth center and the tooth root, material flow in these planes can only be radial. The section plane through the tooth center was chosen for the simulations of the cold forging process of a differential side gear, as it is performed by an ERC / NSM member company. A simulation represents the forming of a truncated cone. If the actual face cone cross section was used, shown in Fig.1, the billet length would become unrealistically long in order to obtain filling of the cone [8]. It was therefore decided to use an averaged cone geometry. By changing the angle of the cone, but retaining the diameters at the top and bottom, as shown in Fig.1, the appropriate volume was achieved. A solid modeler was used to calculate the volume. Thus, the simulation could be performed using the original billet length.The simulation results were evaluated by comparing the predicted metal flow with the actual cold forged sequence and with layered plasticine experiments.

\section{Increasing punch life in cold forging of bevel gears}

In the production of complex cold forgings using multiple actions, the maximum allowable punch load is often a limiting factor. In many applications punch loads are very high to achieve complete die filling. Such an example is the cold forging process of the differential side gear. During production punches must be changed due to low cycle fatigue crack initiation and growth in the upper punch. The 2-D process simulation with DEFORM was performed. The normal and shear component of the contact stress distribution at the end of the stroke was used as load input data for the subsequent stress. Very high stresses are observed at the lower punch corner(first peak)and in the upper fillet radius(second peak). The equivalent stress distribution near the punch tip, resulting from the normal and shear components of the contact stress distribution. The values are obtained by an elastic-plastic stress analysis using the FE code ABAQUS. Very high stresses occur at the upper corner of the punch up. Assuming a yield strength of $2500 \mathrm{MPa}$ for an AISI M2 punch steel hardened to $65 \sim 67 \mathrm{HRC}$, the zone below the No.10 contour line would experience small amounts of plastic straining, resulting in gradual crack initiation and propagation. The area experiencing plastic strain agrees with the region of the punch that fails during production.

\section{Simulation of the rear lower arm forging}

Here, just the simulation of the bust step is introduced. Although the actual deformation of the bust step is 3-dimensional, by supposing the branch arms are symmetrical about center line $\mathrm{O}$, as shown in Fig.2, it may be simplified as 2-dimensional problem. Hence, the deformation process may be devided into two stages: the first one, beginning from the upset to the moment when the material contacts the die wall, is considered as axisymmetric deformation stage; the second, from the above moment to the end of deformation, is supposed as plane-strain stage. In axisymmetric deformation stage, the characteristic of deformation is free upset. When the axisymmetric deformation stage finished, the billet becomes an inscribed cylinder contacting die wall, the dotted line as shown in Fig.2. Suppose the three branch arms of the rear lower arm forging are symmetric, then the plane OD, OE, and OF are neutral boundaries. Metal in the fan-shaped column, EOF will flow into the branch A. In an analogous manner, metal in fan-shaped columns 
FOD and DOE will flow into the branch arms $\mathrm{B}$ and $\mathrm{C}$ respectively. Here only the deformation of the branch $\operatorname{arm} \mathrm{A}$ is analysed.

In plane-strain stage, the deformation characteristic is that metal is extruded toward flank. The line $\mathrm{O}$ (axis Y)is neutral boundary, fixed restraint. When the plane-strain stage starts, metal in fan-shaped column EOF will flow toward die cavity of the branch arm A, as shown in Fig.2. At first, with the FEM-Program of the rigid plastic and rigid visco plasticity, the simulation of the rear lower arm forging was conducted according to initial dies. The results of simulation is that the lap defect occurs on the upper surface of metal near the exit of the expanded channel. In this case, the forging is a failure.

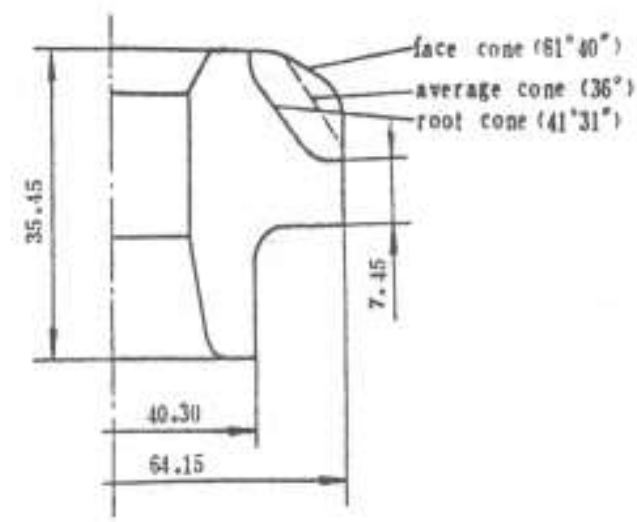

Figure 1. Geometry of differential side gear

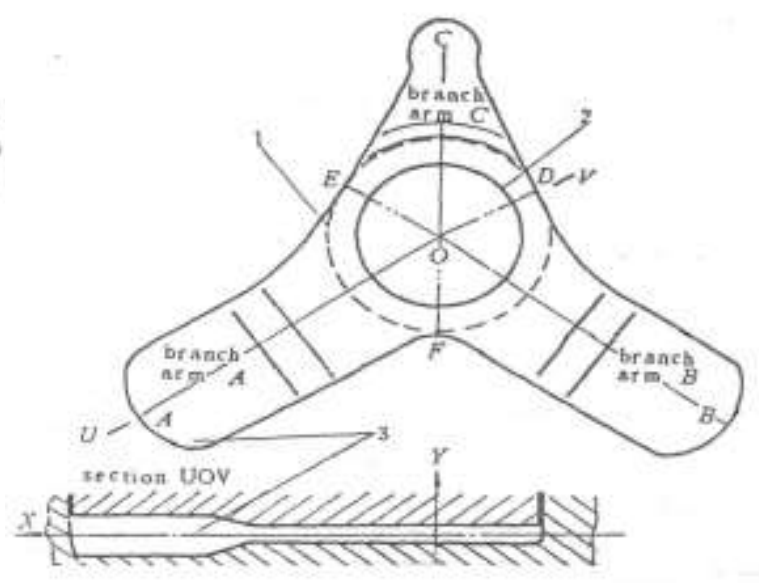

Figure 2. Schemtic illustration of billet and cavity

\section{Conclusions}

The synthetical symptoms of bevel gear is concluded. Using wavelet and mixed data merge does the intelligence diagnosis to the defect of bevel gear, which is integrated with data , characteristic, decision grate and nerve network . A model of wavelet neural network is constructed. In order to reduce no confirm of defect analysis, the excellent diagnosis way is studied with the information of many sources fill and redundant. The result is given that using mixed data merge may raise tolerate character with the help of many sources fill and redundant,and using wavelet and mixed data merge does the effective diagnosis of bevel gear.

\section{References}

[1] T. Tröster and W. Rostek, Advanced Hot Forming, in proceedings from The International Conference New Development in Sheet Metal Forming Technology, Stuttgart, Germany (2004),p.49-63. [2] R. Kolleck et al, Hot Forming and Cold Forming Two Complementary Processes for Lightweight Auto Bodies, in proceedings from The International Conference New Development in Sheet Metal Forming Technology, Stuttgart, Germany (2004), p. 235-244.

[3] Altan T, Ohs, Gegel. Metal Forming:Fundamental and Applications. American Society for Metal.Metal Park. 1983.

[4] Nakagawa T, Wei J. Laser Stereolithography and Its Application to Forming Industries. .Proc.of 4th Ictp.1993.

[5] Dean T.A.Progress in Net-Shape Forging.Proc.of 4th Ictp.1993.

[6] Sheljaskovs. Warm Forging in Comparison with Hot and Cold Forging.Proc.of 4th Ictp. 1993.

[7] Schmoeckel D, et al. State of development and range of application of warm forging. 1992.

[8] SchmoeckelD, et al. Warmforging as an alternative to cold extrusion and hot forging in production of large lot sizes. 1993. 